\section{GP243 NEONATAL THERAPEUTIC HYPOTHERMIA IN IRELAND, 2016-2017}

${ }^{1}$ Sarah Meaney, 2,3 Siobhan Horkan*, 4,2,5Julie Mc Ginley, 'Paul Corcoran, ${ }^{1}$ Richard Green, 2,5John Murphy, Neonatal Therapeutic Hypothermia Working Group ${ }^{2} .{ }^{1}$ NPEC, UCC, Cork, Ireland; ${ }^{2}$ RCPI, Dublin, Ireland; ${ }^{3}$ Portiuncula University Hospital, Ballinasloe, Ireland; ${ }^{4}$ NWIHP, Dublin, Ireland; ${ }^{5}$ National Maternity Hospital, Dublin, Ireland

10.1136/archdischild-2019-epa.302

Before or during birth, a small number of infants experience reduced oxygen or blood supply. After birth, some of these infants will show abnormal neurological behaviour, diagnosed as hypoxic-ischemic encephalopathy (HIE). The consequences of HIE for the infant, their family and the wider society are considerable. Therapeutic hypothermia $(\mathrm{TH})$ is a therapy which involves cooling an infant to a targeted temperature below an infants' normal core body temperature and is now the standard treatment for term infants (babies born after 36 completed weeks of gestation) with moderate to severe HIE. Research has demonstrated $\mathrm{TH}$ reduces the rate of death, severe disability and lifelong cerebral palsy for infants born with HIE.

A standardised dataset was developed to collect detailed clinical data on the maternal, infant and clinical characteristics associated with $\mathrm{TH}$. In Ireland, $\mathrm{TH}$ is administered in the four tertiary maternity hospitals, whereby infants born in other hospitals requiring this treatment are transferred to one of these four tertiary hospitals. Anonymised data were collected on site in the 19 maternity units/hospitals and neonatal intensive care units or special care baby units (NICU/SCBU) in the Republic of Ireland on all infants requiring $\mathrm{TH}$ between 1 January 2016 and 31 December 2017.

Over the two year period, 140 infants required $\mathrm{TH}$ which suggests that one in 900 infants born in Ireland during 2016/ 2017 required TH. Nulliparous women accounted for $60 \%$ of the TH cohort $(n=84)$. Of the women whose infants underwent TH, 18.6\% $(n=26)$ experienced maternal pyrexia during labour and $10 \%$ of women had a prolonged rupture of membranes $(n=14)$. Less than $2 \%$ of mothers had an elective caesarean section. At one minute after birth 79.7\%; of infants had an Apgar score between zero and three $(n=110)$. Almost all infants required resuscitation at birth $(95 \% ; n=133$ of 140 ), with $59.3 \%$ of infants needing intubation $(n=83)$. Over the two year period, $60 \%(n=84)$ were born in a tertiary hospital with $40 \%(n=56)$ of infants requiring transfer from a regional or local hospital. The survival rate for the $\mathrm{TH}$ cohort was $88 \%$, as 17 of the 140 infants died.

The findings of this audit illustrate the logistical challenges faced with the delivery of a high acuity, uncommon treatment that has to be delivered on short notice. These findings also highlighted that there was an overrepresentation of complications preceding and during the delivery of the infants requiring $\mathrm{TH}$.

\section{GP244 IMPLEMENTING DEVELOPMENTALLY-FRIENDLY INTERVENTIONS IN A NEONATAL UNIT- 10 YEARS ON}

${ }^{1}$ Ambalika Das, 'Baljit Wilkhu, ${ }^{2}$ Alpa Jivanbhaga*. 'Queens Hospital, Barking, Havering and Redbridge University Hospital NHS Trust, Romford, UK; ${ }^{2}$ Barking, Havering and Redbridge University Trust Hospitals, London, UK

10.1136/archdischild-2019-epa.303

Background Developmentally-supportive practices are proven to improve neuro-developmental outcome of preterm infants vulnerable to brain injury. However, such interventions and practices are hard to implement in the highly medicalised intensive care environment of the neonatal unit. We implemented a range of interventions which led to improved outcome over a period of time.

Methods We introduced environmental modification (optimisation of lighting, noise reduction, introduction of quiet period), positioning guidelines, sleep protection (minimising non-essential clinical contact, cluster care, use of incubator covers), pain management (using pain scores, breast-milk, swaddling, containment), breast-milk provision (skin-to-skin, expression, early suckling), early parental interaction(unlimited access, active care involvement) as developmentally-supportive practices. These practices were introduced at different stages keeping in pace and in harmony with change of culture of the unit. Ten years on these practices are fully embedded as routine.

Analysis The drive started in 2008, taking momentum in 2009. We compared key clinical indicators during 2012-13 and 2017-18 to evaluate long-term effect of such intervention. There was no significant change in the unit's clinical practice during this period.

Results Compared to 2012-13, in 2017-18 total invasive ventilator days reduced from 373 days to 255 days, >90th centile LOS reduced from $4.9 \%$ to $3.31 \%$, inpatient breast-milk days increased from $22.45 \%$ to $31.33 \%$, breast-milk at discharge improved from $30.8 \%$ to $56.5 \%$ and breastfeeding at discharge for $<33$ weeks babies increased from $12 \%$ to $39.8 \%$. On feedback, parental involvement in care and satisfaction improved significantly. At 2-year $68 \%$ babies born at $<30$ weeks had normal neuro-developmental outcome.

Conclusion Implementation of developmentally supportive practices when embedded in unit's practice over a length of time improve clinical and developmental outcome.

\section{GP245 THE USE OF A DOCUMENTATION TOOL 'CENTRAL LINE STICKER' AS A QUALITY IMPROVEMENT INITIATIVE TO STANDARDISE THE INSERTION OF PERIPHERAL CENTRAL CATHETERS (PICC) IN A REGIONAL NEONATAL UNIT}

Nosheen Akhtar*, Siobhan Hackett, Siobhan Gormally. Our Lady Of Lourdes Hospital, Drogheda, Ireland

\subsection{6/archdischild-2019-epa.304}

Background In 2014, a retrospective audit of 25 Neonatal central line (CL) insertions in our NICU demonstrated a suboptimal clinical documentation standard together with a low kappa inter-observer variation score (0.519) between Consultant Radiologist and Paediatrician for CL tip location. A quality improvement initiative in the form of a standardised documentation tools 'CL sticker' was introduced in 2014.

Departmental policy stipulated that it was to be inserted into the patient chart for each central line.

Aims To measure compliance with use of ('CL sticker') for insertion of PICC lines.

To measure inter-observer agreement in relation to PICC lines tip position

To compare results with central line audit in 2014 .

Method Retrospective audit of charts of all Neonates with PICC lines in the NICU between August 2015 and August 2018, was performed. The frequency of use of the 'PICC sticker' was documented. Compliance with the 15 PICC documentation criteria was noted.

Kappa score was calculated between radiologist and paediatrician for the PICC position on $\mathrm{x}$-ray. 
Central Line insertion checklist

Date: / / Time: hrs.

Indication:

Catheter Size:

\begin{tabular}{|c|c|c|c|c|c|c|c|}
\hline Type & $\begin{array}{l}\text { Measured } \\
\text { Depth (cms) }\end{array}$ & $\begin{array}{l}\text { inserted to } \\
\text { (cms) }\end{array}$ & $\begin{array}{c}\text { Time of } X- \\
\text { ray }\end{array}$ & $\begin{array}{c}\text { Position on } \\
x \text { ray* }\end{array}$ & No changes ${ }^{* * *}$ & Withdraw*** & $\begin{array}{l}\text { ReX-ray/ } \\
\text { remove*** }\end{array}$ \\
\hline \multicolumn{8}{|l|}{ UAC** } \\
\hline \multicolumn{8}{|l|}{ UVC** } \\
\hline PICC*** & & & & & & & \\
\hline
\end{tabular}

* $X$ rays must always be performed each time the lines are adjusted.

**The recommended position for UAC is between T6-9, UVC in IVC ( $1 \mathrm{~cm}$ above the dlaphragm and not in the atrium) and PICC in SVC/IVC (see guideline).

*** Please document any adjustments made to lines after $x$ ray.

Contrast must be used for PICC position confirmation (see guideline)

Notes

Line taped/secured at

$\mathrm{cm}$

Line suitable for infusion Yes/No Signature: Bleep:

\section{Abstract GP245 Figure 1}

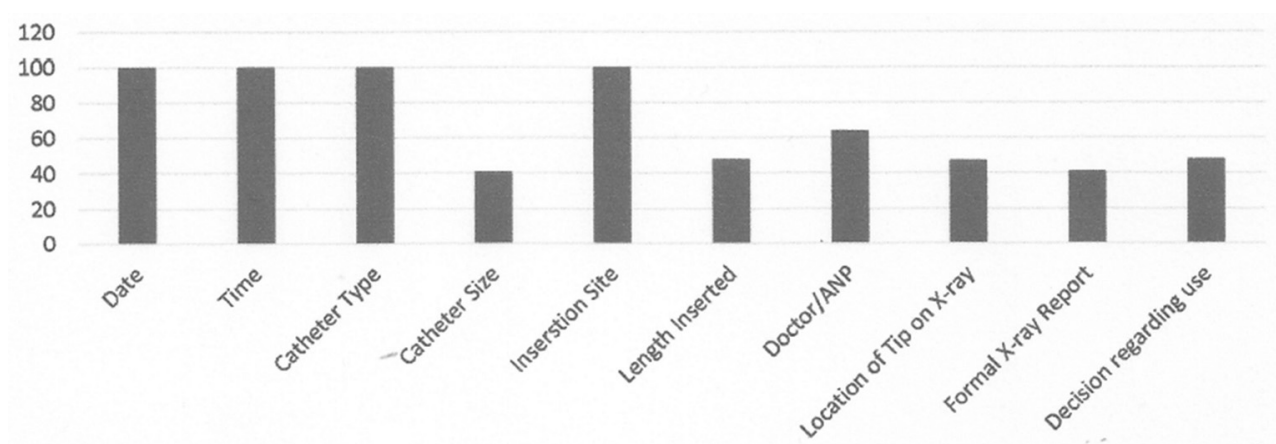

Documentation Criteria 2014 (\%)

- Percentage

Abstract GP245 Figure 2

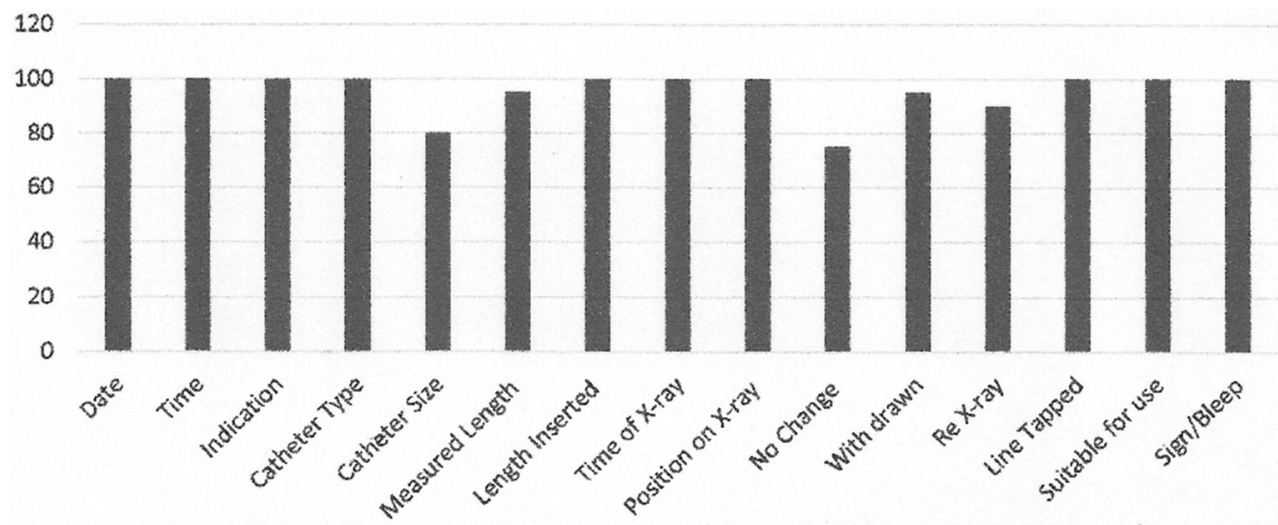

Documentation Criteria 2015-2018 (\%)

- Percentage

\section{Abstract GP245 Figure 3}

